\title{
La Formación Corumbataí y su importancia en la industria cerámica del estado de São Paulo - Brasil
}

\author{
Christofoletti, S.R. ${ }^{\text {I; }}$ Moreno, M.M.T. ${ }^{\text {II }}$ Motta, J.F.M. ${ }^{\text {III }}$

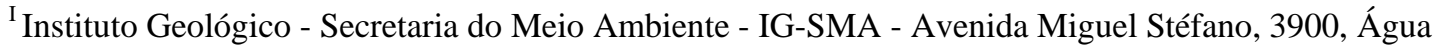 \\ Funda, 04301-903, São Paulo, Brasil. \\ e-mail: sergio@igeologico.sp.gov.br \\ ${ }^{\text {II }}$ Departamento de Petrologia e Metalogenia - DPM/IGCE/UNESP - Campus Santana, Rua 10, 2527, 13500 - \\ 230, Rio Claro, São Paulo, Brasil. \\ e-mail: mmoreno@rc.unesp.br \\ III Divisão de Geologia - Instituto de Pesquisa Tecnológica do Estado de São Paulo - IPT - Cid. Universitária, \\ 05508-901, Avenida Prof. Almeida Prado, 532, São Paulo, Brasil. \\ e-mail: jfmotta@ipt.br
}

\section{RESUMEN}

El desarrollo acelerado del polo cerámico de Santa Gertrudes (provincia de São Paulo) en la década de 90, fue causado principalmente por la localización privilegiada de la materia prima asociada al desarrollo tecnológico, las grandes reservas, el acceso fácil por autopistas que facilitan la distribución de los productos y la proximidad de los grandes centros consumidores. Este desarrollo facilitó que este Polo tuviera un "boom" en el inicio de 2007 en la fase de madurez con una producción de 367 millones de metros cuadrados, transformándose así en el mayor polo cerámico de las Américas. De acuerdo con los estudios geológicos realizados en este trabajo, las rocas de la Formación Corumbataí, origen de la materia prima usada en el polo cerámico, fueron clasificadas en cinco litofacies: maciza, laminada, intercalada I, intercalada II y alterada. Estas litofacies, por sus características mineralógicas y químicas, fueron consideradas arcillas illíticas con porcentaje medio de fundentes. Tomando como referencia las Normas para baldosas cerámicas, las características cerámicas obtenidas en el laboratorio demostraron que la mayoría de la materia prima es compatible con el grupo BIIb (Absorción de agua entre 6 y 10\%).

Palabras claves: industria cerámica, Formación Corumbataí, baldosas, materia prima

\section{The Corumbataí formation and its importance in the ceramic industry of the São Paulo State-Brazil}

\section{ABSTRACT}

The rapid development of the ceramic pole of Santa Gertrudes (São Paulo-Brazil) during the 90's was caused by the privileged localization of the raw material in conjunction with the technological development, large reserves, easy access of the highways helping the draining of the products as well as the proximity of the great consuming centers. This development led the ceramic pole to a "boom" in the decade of 90, entering its maturity phase, producing 367 million $\mathrm{m}^{2}$ in the year of 2007, thus becoming the biggest Ceramic Pole in the American continent. According to the geological studies carried out in this work, the rocks of the Corumbataí Formation, source of the commodity used in the ceramic pole, were classified in five lithofacies: massive, laminated, intercalated I, intercalated II and altered. These lithofacies were considered by their mineralogy and chemistry to be melting clays with predominance of illite. Regarding the characteristic properties, the laboratory results demonstrated the majority of the samples to lie inside the Group BIIb (Absorption of water between 6 and 10\%); it is compatible with the Classification of Ceramic Coverings.

Keywords: ceramic industry, Corumbataí Formation, floor tiles, raw material. 


\section{INTRODUCCIÓN}

En este trabajo fueron identificadas las principales características del Polo Cerámico de Santa Gertrudes (SP-Brasil) enfatizando la estructura de la producción local del sector cerámico y su importancia dentro del contexto nacional. Entre los aspectos abordados, la actividad mineral recibe un enfoque especial, destacándose las características geológicas, tecnológicas, químicas y mineralógicas de la materia prima que es extraída de la formación arcillosa, objetivo de este estudio.

La Formación Corumbataí, de edad Neopermiana, pertenece al Grupo Passa Dois y está inserida en la Cuenca Sedimentar del Paraná. La cuenca abarca un área de 1,600,000 $\mathrm{Km}^{2}$, siendo que su mayor parte está en el territorio brasileño con una extensión de aproximadamente 1,000,000 Km² [1]]. En la región del Polo Cerámico de Santa Gertrudes, esta unidad es formada principalmente por limos (que pueden estar en forma maciza, laminada o intercalada), arcillolitos y areniscas intercaladas con limos arenosos o arcillosos [2]. La distribución de la Formación Corumbataí en el Estado de São Paulo se presenta en la dirección suroeste- noreste pudiendo llegar a la espesura de aproximadamente 30 metros, adelgazando para el Norte hasta anularse en las proximidades del límite con el Estado de Minas Gerais, no pasando los 60 metros en las proximidades de la ciudad de Leme. Su contacto basal con la Formación Iratí es concordante; pero el contacto superior, con la Formación Pirambóia, lo hace mediante discordancia erosiva []ㅡ] (Figura 1).

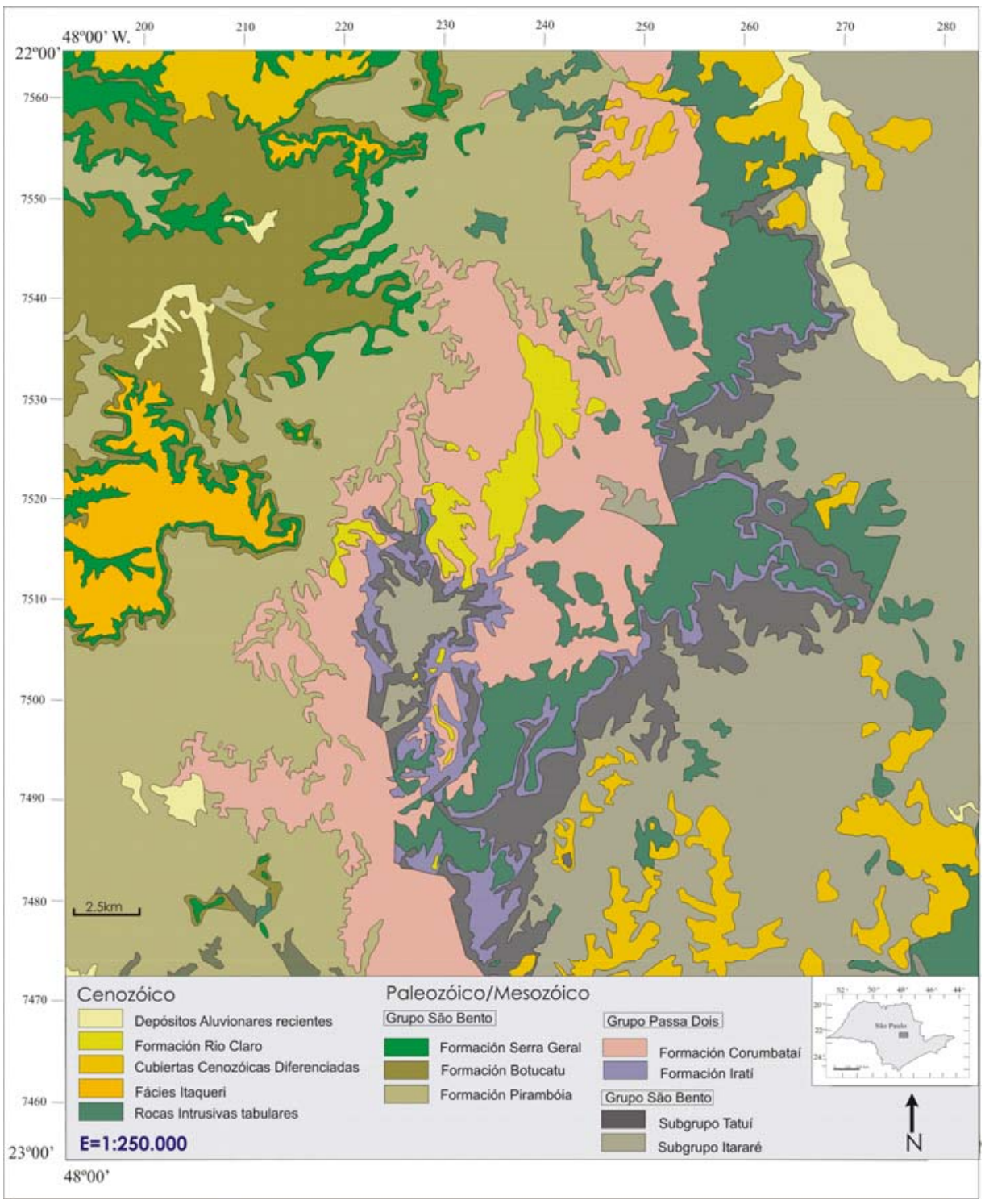

Figura 1: Mapa geológico de la área de estudio, Fuente: CPRM, 2005 [4]]. 


\section{MATERIALES Y MÉTODOS}

Este comprende dos etapas:

a) Compilación de datos generales extraídos de um estúdio realizado em 22 minas, um total de 110 muestras de arcilla, de la región del pólo cerâmico de Santa Gertrudes [2]. Estos datos comprenden: estudio de las rocas de la Formación Corumbataí por medio de mapeo geológico de facies; composición química por Fluorescencia de rayos X en muestra total molida hasta una granulometría menor que ABNT 200 mesh; composición mineralógica por Difracción de rayos X en muestra total y fracción menor que 2 micrometros, y comportamiento tecnológico a través de la caracterización cerámica segundo la norma NBR 13818:1997 [్] en probetas fabricadas a partir de arcilla molida en molino tipo martillo y pasante en tamiz ABNT 30 mesh. Las probetas fueron prensadas en el formato $7 \times 2 \times 1 \mathrm{~cm}$ (5 probetas para cada ensayo) y cocidas en horno eléctrico con temperatura programable ( 1 minuto en la temperatura máxima de $1100^{\circ} \mathrm{C}$ con dos patamares de un minuto en $600^{\circ}$ y $1000^{\circ} \mathrm{C}$ );

b) Compilación de los conocimientos sobre el sector cerámico del Polo Cerámico de Santa Gertrudes y su evolución.

\section{RESULTADOS Y DISCUSIONES}

\subsection{Histórico del Polo Cerámico de Santa Gertrudes}

Santa Gertrudes inició su historia en la industria cerámica entre 1918 y 1930 con la llegada de las primeras familias italianas, que fundaron las primeras alfarerías. Al principio, la producción fue de tejas paulistas y francesas y después de manillas y ladrillos coloniales. Los ladrillos eran baldosas de 30x30 cm, con un peso alrededor de $30 \mathrm{Kg} . \mathrm{m}^{-2}$, extrudidos y cocidos en hornos intermitentes, siendo producidos hasta 1987. Estos productos eran fabricados con la parte más alterada de la Formación Corumbataí.

La molienda se hacía con molino de martillo y la humidificación por goteado, resultando en productos de calidad variable (con defectos de superficie, baja resistencia mecánica, baja adherencia de esmaltes, baja estabilidad dimensional, trincas y grietas) [].

A partir de 1987, hubo un avance tecnológico importante con la implantación del proceso de monococción rápida sobre rodillos cerámicos, haciendo que las empresas invirtieran en esta nueva tecnología para optimizar la calidad y, principalmente, la productividad. La compra de maquinarias modernas y las técnicas avanzadas adquiridas en Italia y España mejoraron significativamente la calidad de los productos.

El aumento de la capacidad productiva de las industrias utilizando la molienda por vía seca ocurrió después de 1994, favorecido por la abundancia de la materia prima disponible, el desarrollo tecnológico, la localización privilegiada y el acceso fácil por las autopistas, facilitando el envío de los productos a los grandes centros consumidores.

\subsection{Perfil y Evolución del Polo Cerámico de Santa Gertrudes}

Actualmente el Polo Cerámico de Santa Gertrudes está constituido por los municipios de Santa Gertrudes, Rio Claro, Araras, Cordeirópolis, Limeira, Ipeúna y Piracicaba, en los cuales se localizan 43 empresas productoras de baldosas cerámicas por vía seca. La producción en 2007 llegó a 367 millones de metros cuadrados, aumentando $12 \%$ en relación a 2006 y con la expectativa de 378 millones de metros cuadrados en 2008 [7]. La capacidad productiva de estas empresas varía entre 2 millones $\mathrm{m}^{2} / \mathrm{mes}$ y 80 millones de $\mathrm{m}^{2} /$ mes, estando $20 \%$ a cargo de las grandes empresas, $40 \%$ de las medias y $40 \%$ de las pequeñas llevándolo a la categoría de mayor polo cerámico de las Américas. (Figura 2).

El Polo Cerámico de Santa Gertrudes reúne geográficamente, abastecedores especializados, universidades, asociaciones de clase, instituciones gubernamentales y otras instituciones que ofrecen educación, información, conocimiento y entretenimiento [8] (Figura 3). Este cuadro tuvo una dinámica evolutiva que pasó de la fase embrionaria (período de 1978 hasta el comienzo de los años noventa) y de crecimiento, encontrándose actualmente en la fase de madurez, la cual tuvo inicio en el año 2,000 (Figura 4). La fase de madurez es caracterizada por la elevada producción, habiendo alcanzando mayoría en el mercado interno y exportando para diversos países del mercado externo. Se espera que en el futuro haya una diversificación de los productos, entre ellos porcelanato y baldosas cerámicas fabricadas por el proceso de via húmeda. 


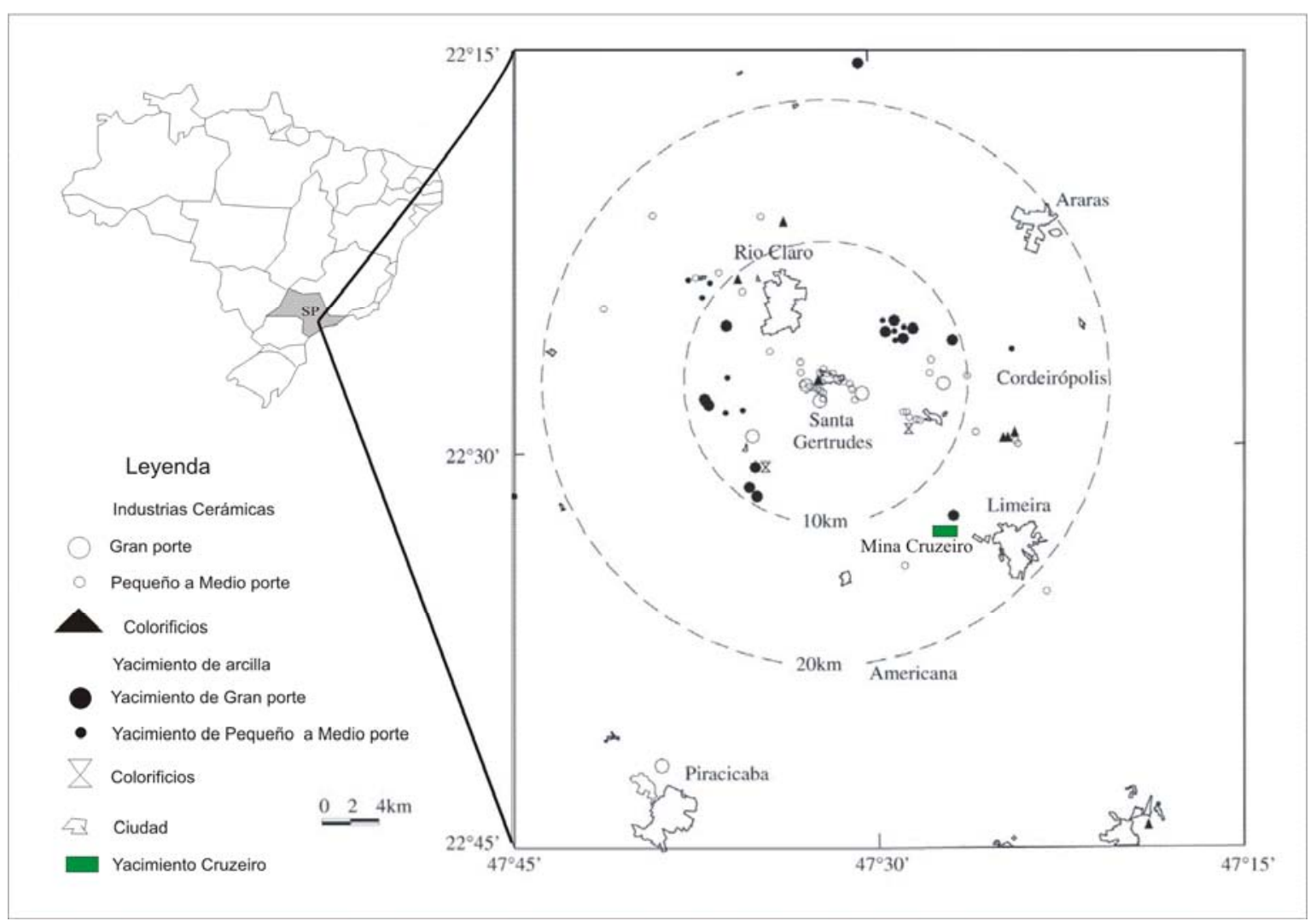

Figura 2: Localización y distribución de las empresas cerámicas y yacimientos de arcilla en el Polo Cerámico de Santa Gertrudis [9].

Una sucesión de innovaciones en el proceso productivo fueron adoptadas, siendo los productores de maquinarias los responsables, por la consolidación de la estructuración. En esta fase comenzaron a instalarse los primeros caloríficos constituyendo el punto de partida para el desarrollo de esmaltes y decoración durante la década de 90. Sumándose a estas características surge el interés de las instituciones de enseñanza (universidades), la modernización de plantas, leyes ambientales más rigurosas y un consumidor más exigente. Es en estas condiciones que el polo cerámico de Santa Gertrudes pasa a competir con el mercado de Santa Catarina (ubicado en la Región Sur) y otros polos del Estado de São Paulo.

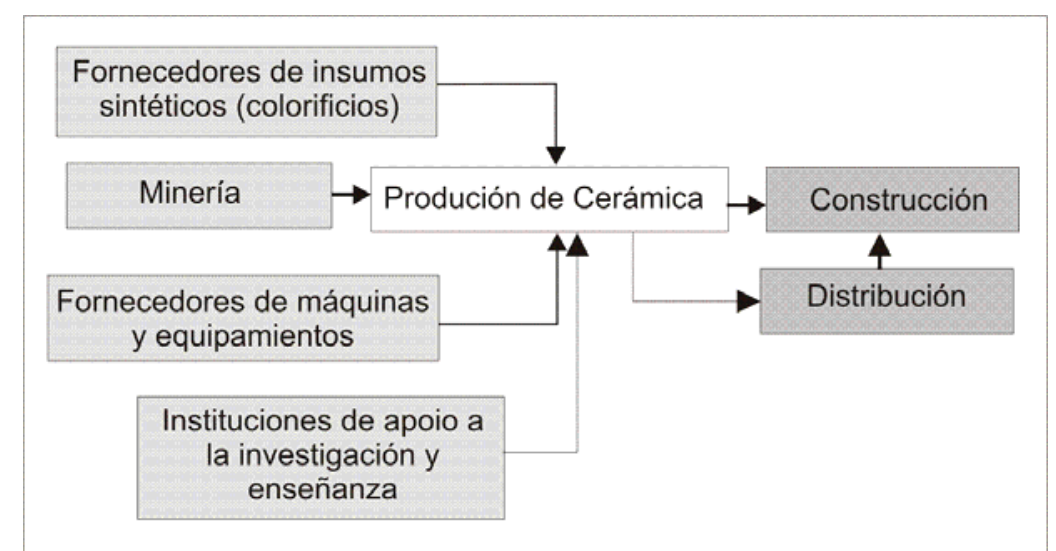

Figura 3: Cadena Productiva de Baldosas Cerámicas en Santa Gertrudes []]. 


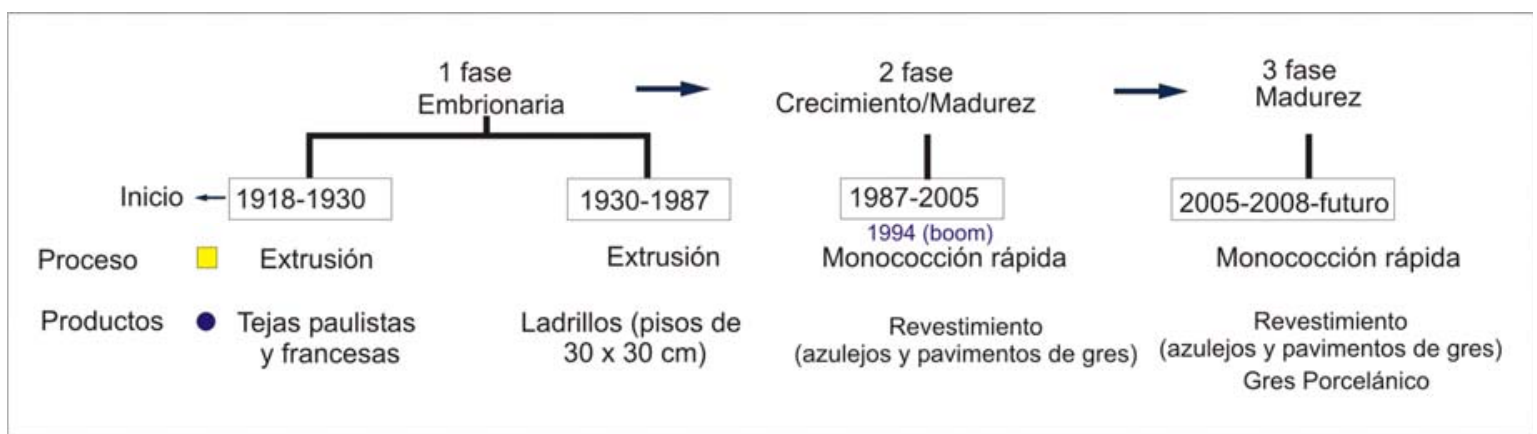

Figura 4: Evolución del Polo Cerámico de Santa Gertrudes (1918-2008).

\subsection{Sistema extractivo de las arcillas en el Polo Cerámico de Santa Gertrudes}

La actividad minera en la región se empezó en 1910 con la exploración de caliza en la Formación Irati. En 1918, la exploración de los suelos resultantes de la alteración de la Formación Corumbataí servía para la fabricación de tejas. Solamente en los años 80, la exploración se hizo efectiva al transformarse en fuente importante de materia prima para la fabricación de baldosas cerámicas. Los yacimientos de arcilla de la Formación Corumbataí se encuentran localizadas de tres maneras distintas: debajo de los depósitos cenozoicos de la Formación Rio Claro y de la Formación Pirambóia (se queda más frecuentemente en la parte noroeste de la región) y debajo de los propios suelos generados por la Formación Corumbataí.

La demanda actual de arcilla local, por las empresas ceramistas del Polo Cerámico de Santa Gertrudes, es del orden de 250,000 toneladas al mes, habiendo una producción adicional de 15-20\% para atender las empresas de otras localidades. Este abastecimiento es efectuado por cerca de 20 yacimientos divididos en tres grupos: los grandes (20-35 mil ton/mes), medios (10-20 mil ton/mes) y pequeños (alrededor de 5 mil ton/mes) [ㅁ] .

Actualmente el área de explotación de la Formación Corumbataí es ejecutada por intereses mineros con aproximadamente 215 títulos para la exploración de los yacimientos. La falta de una gestión en la actividad minera engendra conflictos generalizados con los órganos de fiscalización estadual y federal resultando, algunas veces, en la interrupción de las actividades. Las reservas de arcilla son estimadas en 270 millones de toneladas medidas [10].

El sistema minero del Polo Cerámico de Santa Gertrudes es constituido de varias fases: planeamiento que incluye la prospección e investigación minera, viabilidad técnica y económica de la labra (planeamiento de labra, instalación del yacimiento, preparación de la infraestructura, acceso, compra de máquinas y herramientas), operación (retirada de la vegetación y del suelo, desmonte, cargamento y preparación) y recuperación ambiental de la área degradada. Por otro lado, el proceso cerámico incluye la preparación de la masa, prensado, secado, esmaltado y cocción. En la (Figura 5) son mostradas estas etapas.

\subsection{Características de los Depósitos}

Las minas de arcilla en actividad en el Polo Cerámico de Santa Gertrudes, tienen espesores que varían desde 10 a 45 metros, cambiando sus litofacies tanto vertical como lateralmente. Una sección estratigráfica del yacimiento Cruzeiro, que representa todas las facies identificadas en los perfiles de la región es demostrado en la Figura 6.

Este yacimiento se caracteriza por presentar tendencias de ambiente marino de plataforma y una superposición granocreciente ascendente que es acompañada por aumento en la cantidad de arena. Esta superposición fue iniciada por una sedimentación más profunda, sometida a los procesos de marea de baja energía o decantación (litofacies maciza), que después pasó para dominios de marea de baja energía en un nivel más raso, demostrado por laminaciones incipientes (litofacies laminada), terminando en las partes más rasas, que son dominadas por facies más arenosas (litofacies intercalada). Estas son formadas por procesos de alta energía y, a veces, por las ondas. Según los criterios de reconocimiento de facies basados en la descripción de afloramientos y mapeo geológico [2]], las arcillas de la Formación Corumbataí fueron clasificadas en cinco litofacies: maciza, laminada, intercalada I, intercalada II y alterada (Tabla 1). Estas litofacies, de acuerdo con su semejanza, fueron divididas en dos grandes asociaciones: limo arcillosa y limo arenosa. 


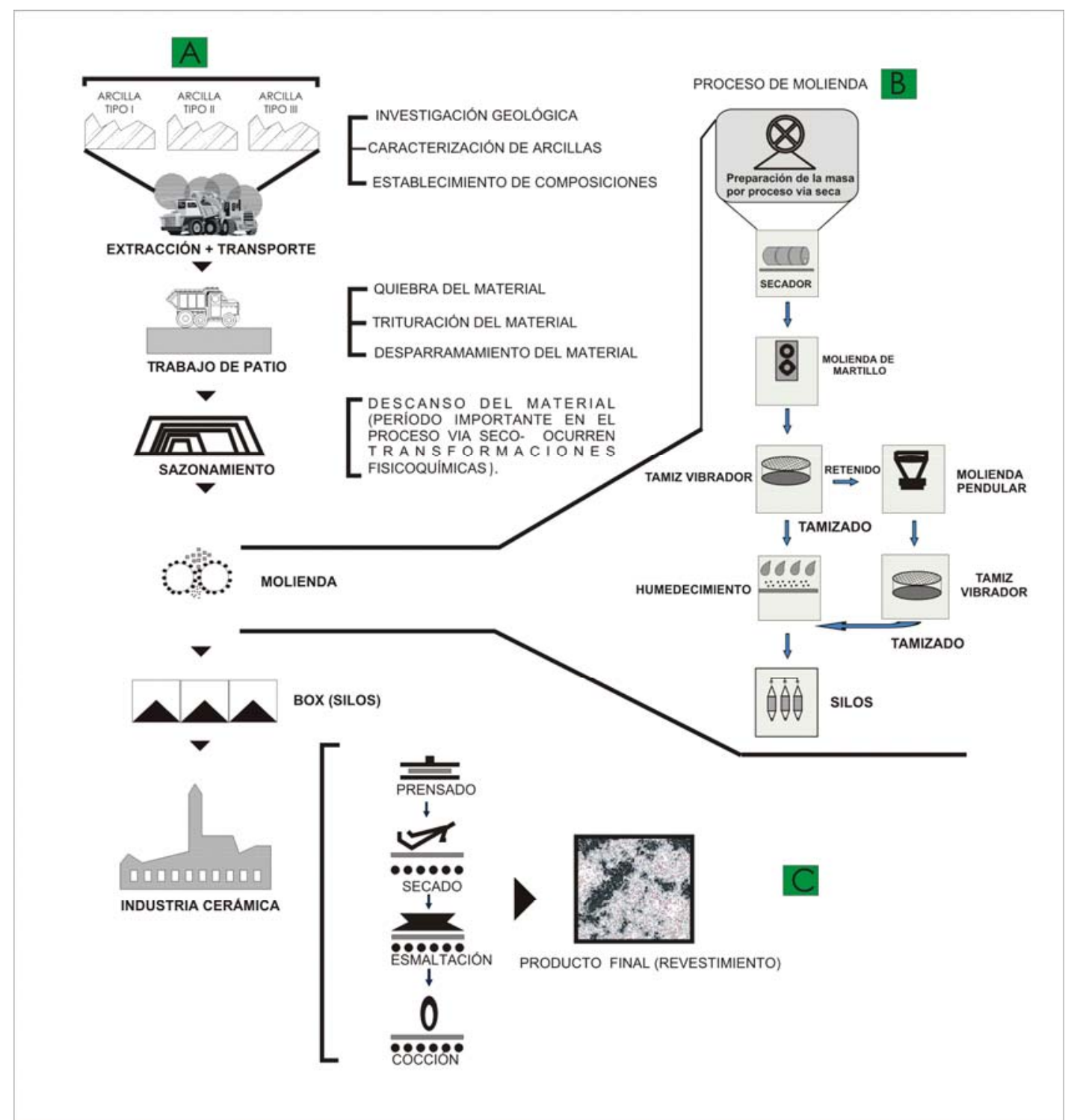

Figura 5: Etapas del proceso productivo del sector minerocerámico de Santa Gertrudes [2] ]. Obs. A=sistema minero, $\mathrm{B}=$ proceso de molienda y $\mathrm{C}=$ proceso industrial.

La asociación limo arcillosa está formada por las litofacies maciza, laminada y alterada (esta puede ser encontrada en todo el perfil de la unidad, dependiendo de la intemperización. Esta asociación es la más representativa en el área estudiada y está ubicada en la base de la Formación Corumbataí. Tiene como estructuras sedimentarias principales laminación incipiente y maciza, color morado grisáceo y granulación arcillosa. En esta asociación predominan los procesos de marea de baja energía o decantación, formando la estructura maciza y laminación plano paralela incipiente dada por arena de granulación muy fina.

La asociación limo arenosa está representada por las litofacies intercalada I y intercalada II y se localiza en la cumbre de la Formación Corumbataí. Estas litofacies son constituidas por intercalaciones rítmicas de limos arcillosos de color morado rojizo, algunas veces intercalados con limos arenosos y otras con areniscas finas. Esas intercalaciones rítmicas son generadas por procesos que van desde la decantación intercalada con marea, hasta las tempestades medias a dístales generando varias estructuras características del tipo de sedimentación. 


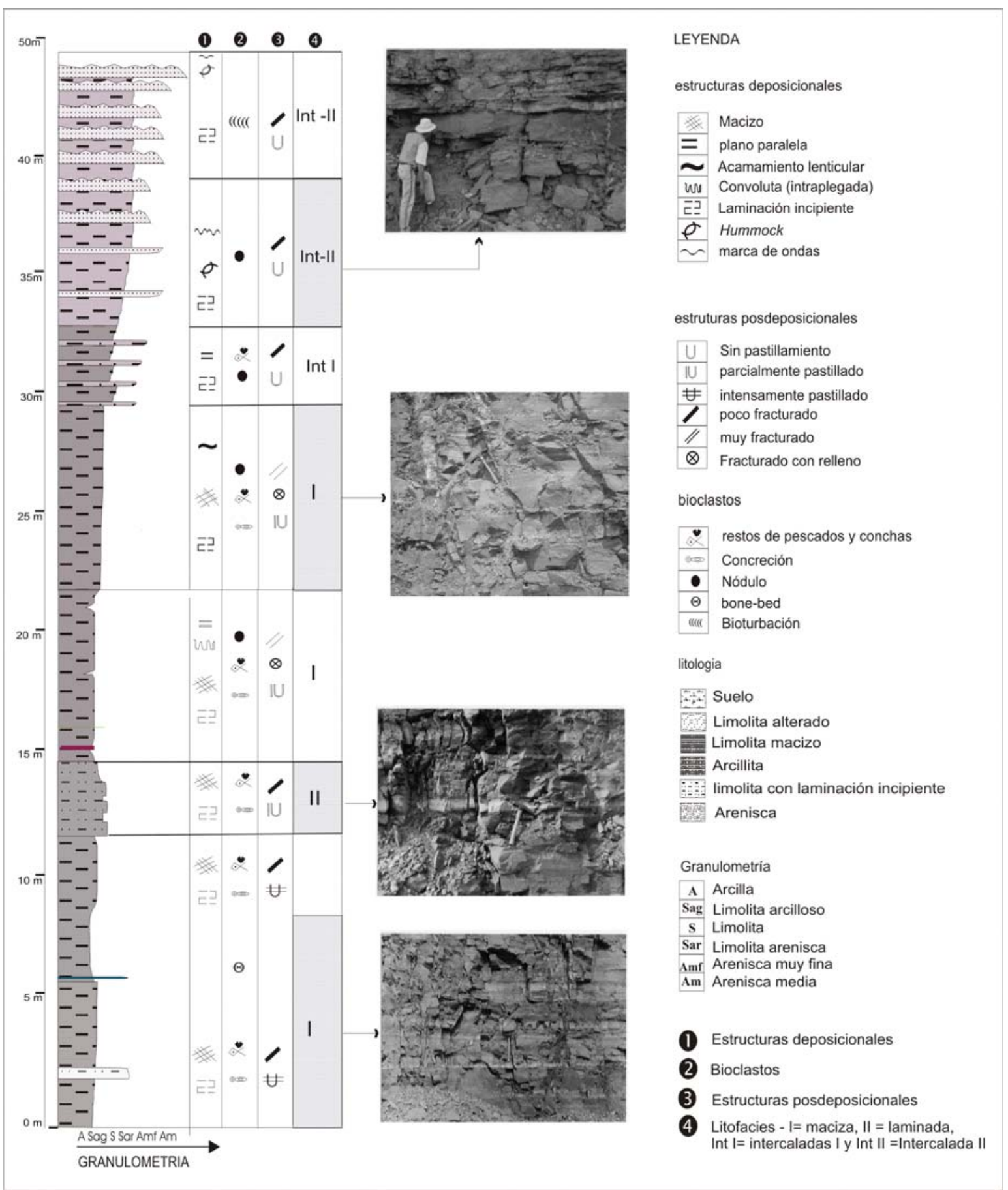

Figura 6: Columna Estratigráfica del yacimiento Cruzeiro-Limeira-SP. 
Tabla 1: Principales Litofacies cerámicas

\begin{tabular}{|c|c|c|c|c|}
\hline \multirow[t]{2}{*}{ Litofacies } & \multicolumn{4}{|c|}{ Características } \\
\hline & Litología & Partículas & Estructura & Color \\
\hline \multicolumn{5}{|c|}{ Asociación Limo Arcillosa } \\
\hline Maciza & Limo & arcilloso a medio & Maciza & $\begin{array}{c}\text { morado, rojizo, } \\
\text { verde, amarillo, gris. }\end{array}$ \\
\hline Laminada & Limo & arcilloso a medio & $\begin{array}{l}\text { laminación plano- } \\
\text { paralela incipiente }\end{array}$ & $\begin{array}{c}\text { morado, rojizo, } \\
\text { verde, amarillo, gris. }\end{array}$ \\
\hline Alterada & Limo & arcilloso & sin estructuras & $\begin{array}{c}\text { blanqueado, rojizo, } \\
\text { morado, gris. }\end{array}$ \\
\hline \multicolumn{5}{|c|}{ Asociación Limo Arenoso } \\
\hline \multirow[t]{2}{*}{ Intercalada I } & Limo & arenoso a medio & \multirow[t]{2}{*}{ Ritmito } & \multirow[t]{2}{*}{ morado rojizo } \\
\hline & Limo & arcilloso & & \\
\hline \multirow[t]{2}{*}{ Intercalada II } & Limo & $\begin{array}{l}\text { arcilloso, arenoso o } \\
\text { medio }\end{array}$ & \multirow[t]{2}{*}{ Ritmito } & morado rojizo \\
\hline & Arenito & $\begin{array}{l}\text { muito fino, médio y } \\
\text { grueso }\end{array}$ & & blanco \\
\hline
\end{tabular}

\subsection{Características Químicas y Mineralógicas}

A partir de los datos del análisis químico de los elementos mayores (aquellos que suman aproximadamente 99,9\%) fueron seleccionados, para establecer relaciones, los óxidos de los elementos considerados fundentes $\left(\mathrm{Na}_{2} \mathrm{O}, \mathrm{K}_{2} \mathrm{O}, \mathrm{CaO}, \mathrm{MgO}\right)$ y los óxidos de los elementos refractarios $\left(\mathrm{SiO}_{2} \mathrm{y} \mathrm{Al}_{2} \mathrm{O}_{3}\right)$, además del óxido de hierro. Estas informaciones se encuentran en la Tabla 2.

El $\mathrm{Fe}_{2} \mathrm{O}_{3}$ exhibe valores medios del orden de 5,3\% para la litofacies laminada. La litofacies intercalada II fue la que presentó características más fundentes y el menor contenido de refractarios, encontrándose valores medios de $14,0 \%$ para el $\mathrm{Al}_{2} \mathrm{O}_{3}$ y de $64,4 \%$ para el $\mathrm{SiO}_{2}$. Excepto las litofacies alterada y la intercalada II, las demás litofacies (maciza, laminada e intercalada I) presentaron cantidades semejantes de fundentes. La litofacies alterada está empobrecida en estos elementos debido a la intemperización y la litofacies intercalada II se destacó de las demás por las mayores cantidades de $\mathrm{CaO}$ y $\mathrm{MgO}$, lo que constituye un indicativo de mayor contenido de carbonatos.

El mineral arcilloso dominante es la illita constatado por análisis comparativa de los gráficos de difracción de la muestra total e por otros autores $[\underline{11}, \underline{12}]$ estando también presentes, cloritas, esmectitas, estratificados regulares e irregulares y, en las litofacies alteradas, la caolinita. Otros minerales encontrados son, por orden decreciente de abundancia: cuarzo, albita, calcita, hematites y dolomita, Tabla 3.

Tabla 2: Elementos mayores. Obs. DESVPAD= Desvío Padrón

\begin{tabular}{|c|c|c|c|c|c|}
\hline \multirow{2}{*}{$\begin{array}{l}\text { Litofacies } \\
\text { Cerámicas }\end{array}$} & \multicolumn{5}{|c|}{ Elementos Mayores en \% } \\
\hline & $\mathrm{Na}_{2} \mathrm{O}+\mathrm{K}_{2} \mathrm{O}$ & $\mathrm{CaO}+\mathrm{MgO}$ & $\mathrm{SiO}_{2}$ & $\mathbf{A l}_{2} \mathbf{O}_{3}$ & $\mathrm{Fe}_{2} \mathrm{O}_{3}$ \\
\hline \multicolumn{6}{|c|}{ Maciza } \\
\hline MEDIA & 3,9 & 2,6 & 67,7 & 14,1 & 5,8 \\
\hline DESVPAD & 1,3 & 1,6 & 2,5 & 1,8 & 1,6 \\
\hline \multicolumn{6}{|c|}{ Laminada } \\
\hline MEDIA & 4,2 & 3,0 & 67,0 & 14,7 & 5,3 \\
\hline DESVPAD & 1,3 & 1,5 & 2,4 & 1,6 & 0,7 \\
\hline \multicolumn{6}{|c|}{ Intercalada I } \\
\hline MEDIA & 3,5 & 2,9 & 67,4 & 15,0 & 4,6 \\
\hline DESVPAD & 1,5 & 3,6 & 3,5 & 2,5 & 1,2 \\
\hline \multicolumn{6}{|c|}{ Intercalada II } \\
\hline MEDIA & 4,3 & 5,4 & 64,4 & 14,0 & 5,0 \\
\hline DESVPAD & 1,4 & 3,8 & 3,10 & 3,4 & 1,3 \\
\hline \multicolumn{6}{|c|}{ Alterada } \\
\hline MEDIA & 2,9 & 1,8 & 64,8 & 17 & 6,0 \\
\hline DESVPAD & 1,2 & 0,5 & 4,0 & 2,7 & 1,4 \\
\hline
\end{tabular}


Tabla 3: Mineralogía de las rocas de la formación Corumbataí.

\begin{tabular}{cc}
\hline $\begin{array}{c}\text { Litofacies } \\
\text { Cerámicas }\end{array}$ & Mineralogía \\
\hline Maciza & Argilominerales y minerales \\
\hline Laminada & illita, clorita, cuarzo, hematita y calcita \\
Intercalada I & illita, clorita, montmorillonita y cuarzo clorita, caolinita, montmorillonita, cuarzo, albita, hematita, calcita y \\
dolomita
\end{tabular}

\subsection{Características Cerámicas}

Con el fin de evaluar los datos se tomó como referencia, la Norma NBR 13818 [ㅁ] que permitió clasificar la mayoría de las argilas dentro del Grupo BIIb (6-10\% de absorción de agua) para probetas prensadas y quemadas a $1100^{\circ} \mathrm{C}$, excepto algunas muestras de las litofacies intercalada I, intercalada II y alterada que dieron valores mayores de 10\% (grupo BIII poroso, 10-20\% de absorción de agua), Tabla 4.

Los valores medios de tensión de ruptura a la flexión (TRF) oscilaron entre $209 \pm 73 \mathrm{Kgf}_{\mathrm{cm}} \mathrm{cm}^{-2}$ para las muestras de la litofacies Intercalada I y $265 \pm 86 \mathrm{Kgf.cm}^{-2}$ para la laminada. Los mayores valores de contracción linear de cocción fueron obtenidos en las litofacies maciza y alterada (8,1\%) cambiando la masa específica aparente (MEA) desde 1,9 hasta 2,1 g. $\mathrm{cm}^{-3}$, en las litofacies intercalada II y laminada, respectivamente.

Tabla 4: Resultados Cerámicos en probetas prensadas y cocidas a $1100^{\circ} \mathrm{C}$. DESVPAD= Desvío Padrón, TRF=tensión de ruptura a la flexión, $\mathrm{AA}=$ absorción de agua, $\mathrm{PA}=$ porosidad aparente, $\mathrm{RLQ}=$ contracción lineal de cocido, MEA=masa específica aparente e PPC=pérdida por calcinación.

\begin{tabular}{|c|c|c|c|c|c|c|}
\hline \multirow[t]{2}{*}{ Litofacies } & \multicolumn{6}{|c|}{ Características cerámicas } \\
\hline & TRF $\left(\mathrm{kgf} / \mathrm{cm}^{2}\right)$ & AA\% & PA\% & RLQ\% & MEA\% & PPC\% \\
\hline \multicolumn{7}{|l|}{ Maciza } \\
\hline MEDIA & 261 & 7,8 & 14,6 & 8,1 & 2,1 & 5,4 \\
\hline DESVPAD & 59 & 6,2 & 9,6 & 1,8 & 0,3 & 2,5 \\
\hline \multicolumn{7}{|c|}{ Laminada } \\
\hline MEDIA & 265 & 6,5 & 11,6 & 7,2 & 2,1 & 4,9 \\
\hline DESVPAD & 86 & 4,9 & 8,3 & 2,6 & 0,2 & 2,5 \\
\hline \multicolumn{7}{|c|}{ Intercalada I } \\
\hline MEDIA & 209 & 12 & 21,5 & 5,5 & 1,9 & 5,3 \\
\hline DESVPAD & 73 & 6,6 & 10,1 & 2,5 & 0,2 & 2,0 \\
\hline \multicolumn{7}{|c|}{ Intercalada II } \\
\hline MEDIA & 259 & 10,0 & 18,8 & 7,0 & 1,9 & 7,0 \\
\hline DESVPAD & 104 & 7,4 & 11,7 & 2,6 & 0,3 & 4,4 \\
\hline \multicolumn{7}{|c|}{ Alterada } \\
\hline MEDIA & 233 & 9,7 & 19,6 & 8,1 & 2,0 & 6,0 \\
\hline DESVPAD & 75 & 5,3 & 8,9 & 2,6 & 0,2 & 1,0 \\
\hline
\end{tabular}

Las litofacies maciza y laminada presentan mejor sinterización en las condiciones ensayadas debido a que el feldespato (dominantemente sódico) está constituido por granos muy finos, que asociados a los de illita favorecen la reactividad entre los compuestos. Por otro lado, la litofacies alterada, a pesar de formar muchos finos durante la molienda, tiene menor cantidad de elementos alcalinos, pues estos fueron parcialmente movidos por intemperización, necesitando así, mayor temperatura de cocción para adquirir el mismo grado de sinterización que las litofacies maciza y laminada. En las litofacies intercaladas I e II, la presencia de material más arenoso en la primera (encontrada en las litofacies superiores Intercalada I e Intercalada II, Figura 6) comprometió la compactación de las probetas, causando deficiencia en la ocupación de los espacios vacíos por los granos menores. En la intercalada II el mayor contenido de carbonatos (5,4\% de $\mathrm{CaO}+\mathrm{MgO}$ ) contribuyó para la mayor porosidad. 
En los ensayos cerámicos, el factor tamaño de grano de prensado fue importante, separando las litofacies más arcillosas (maciza y laminada) de las otras, influenciado principalmente por el grado de alteración intempérica que permitió mayor desagregación de los granos naturales durante la molienda [11], lo que no vale para la maciza. La litofacies intercalada I presentó absorción de agua (AA) alta porque tiene mayor porcentaje de arena de granulometría fina, encontrada en las litofacies superiores Intercalada I e Intercalada II (Figura 6) que actúa como estructurador, dejando las probetas mas grandes.

De acuerdo con las características tecnológicas, todas las arcillas de la Formación Corumbataí en el área estudiada tienen potencial para ser usadas como materia prima en la industria de baldosas, como indican los resultados de los ensayos cerámicos efectuados y también comprobado por [13], siendo que los niveles alterados son usados en la composición de las masas cerámicas para baldosas, para aumentar la plasticidad y en la cerámica estructural. También son encontrados algunos niveles con exceso de contaminantes, tales como venas de cuarzo, carbonatos y fósiles, los cuales pueden originar defectos cuando no son finamente divididos durante la molienda [14].

\section{CONCLUSIONES}

Desde los años 80 hasta la actualidad, el Polo Cerámico de Santa Gertrudes tuvo un crecimiento más allá de lo esperado, con una producción de 367 millones en el año de 2007 y con la expectativa de 378 millones de metros cuadrados en 2008. Esto representa el 44,6\% de la producción de baldosas en todo Brasil, transformándose en el mayor polo cerámico de las Américas y el segundo mayor del mundo. Varios factores contribuyeron para eso: el gran volumen de materia prima de bajo valor, localización cercana, cambio de proceso (bicocción por monococción rápida), innovación tecnológica e investigación y desarrollo.

La evolución y crecimiento del conjunto productivo del Polo Cerámico de Santa Gertrudes está asociado directamente al avance tecnológico de las máquinas que sustituyeron las tecnologías obsoletas de la fase embrionaria (1918-1987) donde el proceso era simple y barato, con un producto final sin calidad. La materia prima utilizada era la parte alterada de la Formación Corumbataí, siendo que desde 1987 le fue dada una aplicación más específica, iniciándose la fase de crecimiento. Con las innovaciones tecnológicas, las exigencias de los consumidores y la competencia entre fabricantes de baldosas, se espera, para el futuro, que la materia prima de la Formación Corumbataí resulte, por ejemplo, en gres porcelánico o baldosas por vía húmeda, adicionando o no otras materias primas.

Las arcillas de la Formación Corumbataí en la región del estudio, fueron clasificadas en cinco litofacies: maciza, laminada, intercalada I, intercalada II y alterada. Estas litofacies, por semejanzas en sus características, fueron divididas en dos grandes asociaciones: limo arcillosa y limo arenosa. Los resultados de los análisis químicos de los elementos mayores demuestran que son arcillas, con contenido medio de fundentes, desde los óxidos alcalinos $\left(\mathrm{Na}_{2} \mathrm{O}+\mathrm{K}_{2} \mathrm{O}\right)$, entre 2,9 y 4,3\%, y los óxidos alcalinos térreos $(\mathrm{CaO}+\mathrm{MgO})$ entre 1,8 y $5,4 \%$.

Predominan los minerales arcillosos como illita, clorita, caolinita, montmorillonita y estratificaciones de los mismos (principalmente en las litofacies alteradas). Otros minerales como cuarzo, albita, calcita, hematita y dolomita también son encontrados en cantidades variables.

De acuerdo con las características tecnológicas (probetas $2 x 7 \mathrm{~cm}$, cocidas a $1100^{\circ} \mathrm{C}$ y presentando densidad de cocción entre 1,9 y 2,1 g.cm- ${ }^{3}$ ), todas las arcillas de la Formación Corumbataí en el área estudiada tienen potencial para ser usadas como materia prima en la industria de baldosas, como indican los resultados de los ensayos cerámicos efectuados. La mayoría de las muestras se clasificaron dentro del grupo BIIb, con excepción de las litofacies intercaladas I y II, que tienen absorción de agua mayor que 10\%, clasificándose dentro del grupo BIII, por la presencia de mayor cantidad de material con carbonatos y arenoso, respectivamente, identificado en la descripción faciológica.

\section{AGRADECIMENTOS}

Los autores agradecen el apoyo financiero dado por la Fundacão de Amparo à Pesquisa do Estado de São Paulo - FAPESP. A la Dra. Annabel Pérez de Aguilar y a la Dra. Claudia Luciana Varnier que contribuyó en la corrección del texto.

\section{BIBLIOGRAFÍA}

[1] MILLANI, E.J., Evolução Tectôno-Estratigráfica da Bacia do Paraná e seu relacionamento com a Geodinâmica Fanerozóica do Gondwana Sul-Ocidental, Tese de D.Sc., Universidade Federal do Rio Grande do Sul, Rio Grande do Sul, Brasil, 1997. 
[2] CHRISTOFOLETTI, S.R., Um modelo de classificação geológico-tecnológica das argilas da Formação Corumbataí utilizadas nas indústrias do Pólo Cerâmico de Santa Gertrudes, Tese de D.Sc., Instituto de Geociências e Ciências Exatas Universidade Estadual Paulista, Rio Claro, SP, Brasil, 2003.

[3] LANDIM. P.M.B., O Grupo Passa Dois (P) na bacia do Rio Corumbataí (SP), Boletim da Divisão de Geologia e Minas, Rio de Janeiro, DNPM, (252), pp. 1-103, 1970.

[4] PERROTA, M.M., SALVADOR, E.D., LOPES, R.C., D`AGOSTINHO, L.Z., et al., "Mapa geológico do estado de São Paulo”, escala 1:750.000. Programa levantamentos geológicos básicos do Brasil, CPRM, São Paulo, 2005.

[5] ASSOCIAÇÃO BRASILEIRA DE NORMAS TÉCNICAS - ABNT, NBR 13818 - Placas cerâmicas para revestimentos-especificação e métodos de ensaios, Rio de Janeiro, pp. 59-70, 1997.

[6] PIVA, M.A., PACHECO, A.C.B. "O que as indústrias de revestimento cerâmico da região de Santa Gertrudes esperam das instituições de ensino, pesquisa e desenvolvimento”, Revista Cerâmica Industrial, v. 2, n. 5/6, pp. 7-10, Setembro/Dezembro 1997.

[7] ASPACER- Associação Brasileira de Cerâmica, http://www.aspacer.com.br. Acessado em 08 de janeiro de 2009.

[8] MACHADO, S.A., Dinâmica dos Arranjos Produtivos Locais: Um estudo de caso em Santa Gertrudes, a nova capital da cerâmica brasileira, Tese de D.Sc., Escola Politécnica Universidade de São Paulo, São Paulo, SP, 2003.

[9] MOTTA, J.F.M., CHRISTOFOLETTI, S.R., GARCEZ, L., MORENO, M.M.T., CHUCIERATO, G., FLORENCIO, R.V., ZANARDO, A., "Raw materials for ceramic tiles in the Santa Gertrudes pole, Brazil”. Interceram Review, v. 54, n. 3, pp. 90-94, 2005.

[10] DNPM-Departamento Nacional de Produção Mineral, http://www.dnpm.gov.br. Acessado em 24 de abril de 2008.

[11] ROCHA, R.R., Estudo do comportamento reológico de suspensões argilosas da Formação Corumbataí, Dissertação de M.Sc., Instituto de Geociências e Ciências Exatas Universidade Estadual Paulista, Rio Claro, SP, Brasil, 2007.

[12] IBRAHIM, L., Argilominerais da porção basal da Formação Corumbataí na região de Rio Claro, Tese de D.Sc., Instituto de Geociências e Ciências Exatas Universidade Estadual Paulista, Rio Claro, SP, Brasil, 2008.

[13] ROVERI, C.D., ZANARDO, A., MORENO, M.M.T., et al., "Composition varation of the Corumbataí formation and mine locations in the ceramic pole of the Santa Gertrudes-Brazil”, In: anales del IX Worl Congress on Ceramic Tile Quality: Qualice’06, v. 3, pp. 185-188, Castellón, Espanha, Fevereiro 2006.

[14] CHRISTOFOLETTI, S.R., MASSON, M.R., MIJOLARO, W., MORENO, M.M.T., VALARELLI, J.V., "Os principais contaminantes minerais das rochas sedimentares da formação Corumbataí e sua influência na matéria-prima cerâmica”, Revista Cerâmica Industrial, v. 8, n. 1, pp. 35-41, JaneiroFevereiro 2003. 